\title{
Clinical Outcomes and Predictors of Thirty-Day Readmissions of Hypertriglyceridemia-Induced Acute Pancreatitis
}

\author{
Asim Kichloo a, b, Zain El-amir ${ }^{\mathrm{a}}$, Maria Aucar ${ }^{\mathrm{a}}$, Dushyant Singh Dahiya ${ }^{\mathrm{a}, \mathrm{k}}{ }^{\mathbb{C}}$, Mohammad Al-Haddad ${ }^{\mathbb{E}}$, \\ Sailaja Pisipatid ${ }^{d}$, Hassan Beiz ${ }^{\mathrm{a}}$, Gurdeep Singhe, Darshan Gandhif ${ }^{\mathrm{f}}$, Jagmeet Singh ${ }^{\mathrm{g}}$, \\ Patrick Pathappillil $^{\text {h }}$, Haseeb Mohideen ${ }^{\mathrm{i}}$, Hafeez Shaka ${ }^{\mathrm{j}}$
}

\begin{abstract}
Background: Hypertriglyceridemia (HTG) is a well-established cause of acute pancreatitis often leading to significant morbidity, mortality, and healthcare burden. This study aimed to describe the rate, reasons, and predictors of HTG-induced acute pancreatitis (HTG-AP) in the USA.

Methods: This retrospective study analyzed the Nationwide Readmissions Database (NRD) for 2018 to determine all adults ( $\geq 18$ years) readmitted within 30 days of an index hospitalization of HTGAP. Hospitalization characteristics and adverse outcomes for 30-day readmissions were highlighted and compared with index admissions of HTG-AP. Furthermore, independent predictors for 30-day readmissions of HTG-AP were also identified. P values $\leq 0.05$ were considered statistically significant.
\end{abstract}

Results: In 2018, the rate of 30-day readmission of HTG-AP was noted to be $13.5 \%$. At the time of readmission, AP (45.2\%) was iden-

Manuscript submitted November 15, 2021, accepted December 28, 2021

Published online January 10, 2022

aDepartment of Internal Medicine, Central Michigan University College of Medicine, Saginaw, MI 48603, USA

bDepartment of Internal Medicine, Samaritan Medical Center, Watertown, NY, USA

'Division of Gastroenterology and Hepatology, Department of Internal Medicine, Indiana University School of Medicine, Indianapolis, IN 46202, USA

${ }^{\mathrm{d}}$ Division of Gastroenterology and Hepatology, Mayo Clinic, Scottsdale, AZ 85259 , USA

eDepartment of Medicine and Endocrinology, Our Lady of Lourdes Memorial Hospital, Binghamton, NY, USA

fDepartment of Radiology, Northwestern Memorial Hospital, Northwestern University Feinberg School of Medicine, Chicago, IL 60611, USA

gDepartment of Internal Medicine, Guthrie Robert Packer Hospital, Sayre, PA 18840, USA

hDepartment of Internal Medicine, Creighton University, Phoenix, AZ 85012, USA

iDepartment of Internal Medicine, Indiana University School of Medicine, Indianapolis, IN 46202, USA

jDepartment of Internal Medicine, John H. Stroger, Jr. Hospital of Cook County, Chicago, IL 60612, USA

${ }^{\mathrm{k}}$ Corresponding Author: Dushyant Singh Dahiya, Department of Internal Medicine, Central Michigan University College of Medicine, Saginaw, MI 48603, USA. Email: dush.dahiya@gmail.com

doi: https://doi.org/10.14740/gr1484 tified as the most common principal diagnosis, followed by chronic pancreatitis $(6.3 \%)$ and unspecified sepsis $(4.8 \%)$. Compared to index admissions, 30-day readmissions of HTG-AP had a higher proportion of patients with Charlson Comorbidity Index (CCI) scores $\geq 3$ (48.5\% vs. $33.8 \%, \mathrm{P}<0.001)$. Furthermore, we noted higher rates of inpatient mortality ( $1.7 \%$ vs. $0.7 \%$, odds ratio (OR): $2.55,95 \%$ confidence interval (CI): $1.83-3.57, \mathrm{P}<0.001)$, mean length of stay (LOS) $(5.6$ vs. 4.1 days, OR: $1.5,95 \% \mathrm{CI}: 1.2-1.7, \mathrm{P}<0.001)$, and mean total healthcare charge (THC) (\$56,799 vs. $\$ 36,078$, OR: $18,702,95 \%$ CI: 15,136 - 22,267, P < 0.001) for 30-day readmissions of HTG-AP compared to index admissions. Independent predictors for 30-day allcause readmissions of HTG-AP included hypertension, protein energy malnutrition (PEM), CCI scores $\geq 3$, chronic kidney disease and discharge against medical advice.

Conclusions: AP was the principal diagnosis on presentation in only $45.2 \%$ patients for 30 -day readmissions of HTG-AP. Compared to index admissions, 30-day readmissions of HTG-AP had a higher comorbidity burden, inpatient mortality, mean LOS and mean THC.

Keywords: Readmissions; Hypertriglyceridemia; Acute pancreatitis; Outcomes; Mortality

\section{Introduction}

Hypertriglyceridemia (HTG), characterized by elevated fasting plasma triglycerides, is frequently encountered in routine clinical practice, and may lead to poor clinical outcomes and complications if untreated [1]. Acute pancreatitis (AP) is one of the most serious complications of severe HTG $(\geq 10$ $\mathrm{mmol} / \mathrm{L}(>885 \mathrm{mg} / \mathrm{dL}))[1,2]$. AP is characterized by severe inflammation of the pancreas and may lead to localized necrosis, multi-organ failure and systemic inflammatory response syndrome [3]. From an etiology perspective, after alcoholic and gallstone pancreatitis, HTG is believed to be the third most common cause of AP with an incidence rate of about $2-4 \%$ across the globe $[4,5]$. In the USA, AP is associated with significant mortality which increases with disease severity, and places a substantial burden on healthcare system as it accounts for approximately 275,000 hospitalizations annually with a total attributable healthcare cost of $\$ 2.6$ billion/year [6, 7]. Moreover, in 2016, readmissions secondary to AP were re- 
ported to be as high as $13.5 \%$ within 30 -days of hospital discharge, with higher rates of readmissions noted for patients with idiopathic, alcohol-induced and HTG-induced AP (HTGAP) $[8,9]$. However, there continues to be paucity of data on 30-day readmissions of HTG-AP with limited studies available. Hence, this study was designed to identify demographic characteristics and rates of 30-day readmissions of HTG-AP and compare them to index admissions. Furthermore, we also identified the most common principal diagnoses at the time of readmission and independent predictors of 30-day all-cause readmissions of HTG-AP to identify individuals at the highest risk of readmissions.

\section{Materials and Methods}

\section{Design and data source}

For this national retrospective study, data were derived from the Nationwide Readmissions Database (NRD) for 2018. The NRD is the largest publicly available, all-payer inpatient health care readmission database drawn from the Agency for Healthcare Research and Quality (AHRQ) Healthcare Cost and Utilization Project (HCUP) State Inpatient Databases (SID) [10]. The NRD 2018 contains patient- and hospital-level discharge data from 28 geographically dispersed states in the USA. In the NRD, up to 40 discharge diagnoses and 25 procedures are collected for each patient using the International Classification of Diseases, Tenth Revision, Clinical Modification/Procedure Coding System (ICD-10-CM/PCS). Hospitals are stratified according to ownership control, number of beds, teaching status, urban/rural location, and geographic region. Furthermore, the NRD allows for a weighted analysis to obtain data on $100 \%$ of the US population.

\section{Study population}

This study included all adult ( $\geq 18$ years) hospitalizations within 30 days of an index hospitalization of HTG-AP. As there were no specific ICD10-CM/PCS diagnosis codes for HTG-AP, the study population was obtained after combining clinical diagnosis codes of AP and HTG and excluding AP secondary to all other etiologies such as gall stones, alcohol, and medications from the analysis as established in prior published studies [11,12]. Furthermore, individuals $<18$ years of age and elective hospitalizations were excluded from the analysis. Using unique hospitalization identifiers, index hospitalizations were identified and the subsequent hospitalization within 30 days was tagged as a readmission.

\section{Outcome measures and statistical analysis}

We analyzed the data using Stata ${ }^{\circledR}$ Version 16 software (StataCorp, TX, USA). All analyses were conducted using the weighted samples for national estimates in adjunct with HCUP regulations for using the NRD database. Comorbidities were calculated as proportions of the cohort and the Chi-squared test was used to compare the characteristics between index hospitalizations and readmissions. Comorbidity burden was assessed using Sundararajan's adaptation of the modified Deyo's Charlson comorbidity index [13].The total healthcare charge (THC) was obtained from the cost-to-charge ratio data provided by HCUP [14]. A univariate regression was used to compare readmission mortality, length of stay (LOS), and THC. Furthermore, the univariate Cox regression analysis was performed to identify variables with hazard ratios $<0.20$ for 30-day readmission of HTG-AP to obtain confounders for readmission. Subsequently, a multivariate Cox regression analysis was performed to identify independent predictors for readmissions within 30 days with $\mathrm{P}$ values $<$ 0.05 set as threshold for statistical significance.

\section{Ethical considerations}

The NRD database lacks patient specific identifiers. Hence, this study was exempt from Institutional Review Board (IRB) approval as per our institutional IRB guidelines. Furthermore, the study was conducted in compliance with the ethical standards of the responsible institution on human subjects as well as with the Helsinki Declaration.

\section{Data availability statement}

The NRD is a large, publicly available all-payer inpatient care database in the USA, containing data on more than 18 million hospital stays. Its large sample size provides sufficient data for analysis across hospital types and the study of readmissions for relatively common and uncommon disorders.

\section{Results}

\section{Rate and reasons for 30-day readmissions of HTG-AP}

For the study period, 43,094 index hospitalizations of HTGAP were identified that met the inclusion criteria. The 30-day readmission rate of HTG-AP was noted to be $13.5 \%$. Figure 1 demonstrates the Kaplan-Meier curve for 30-day all-cause readmissions of HTG-AP. At the time of 30-day readmission, AP was identified as the most common (45.2\%) principal diagnosis, followed by chronic pancreatitis $(6.3 \%)$, unspecified sepsis $(4.8 \%)$, acute renal failure (1.6\%), malignant neoplasm of head of pancreas $(1.1 \%)$ and type 2 diabetes mellitus with ketoacidosis $(1.0 \%)$ (Table 1$)$.

\section{Hospitalization characteristics for index and 30-day read- missions of HTG-AP}

We did not find a statistically significant difference in mean age and gender distribution for 30-day readmissions of HTGAP (Table 2). However, 30-day readmissions of HTG-AP had a higher proportion of patients with Charlson comorbidity in- 


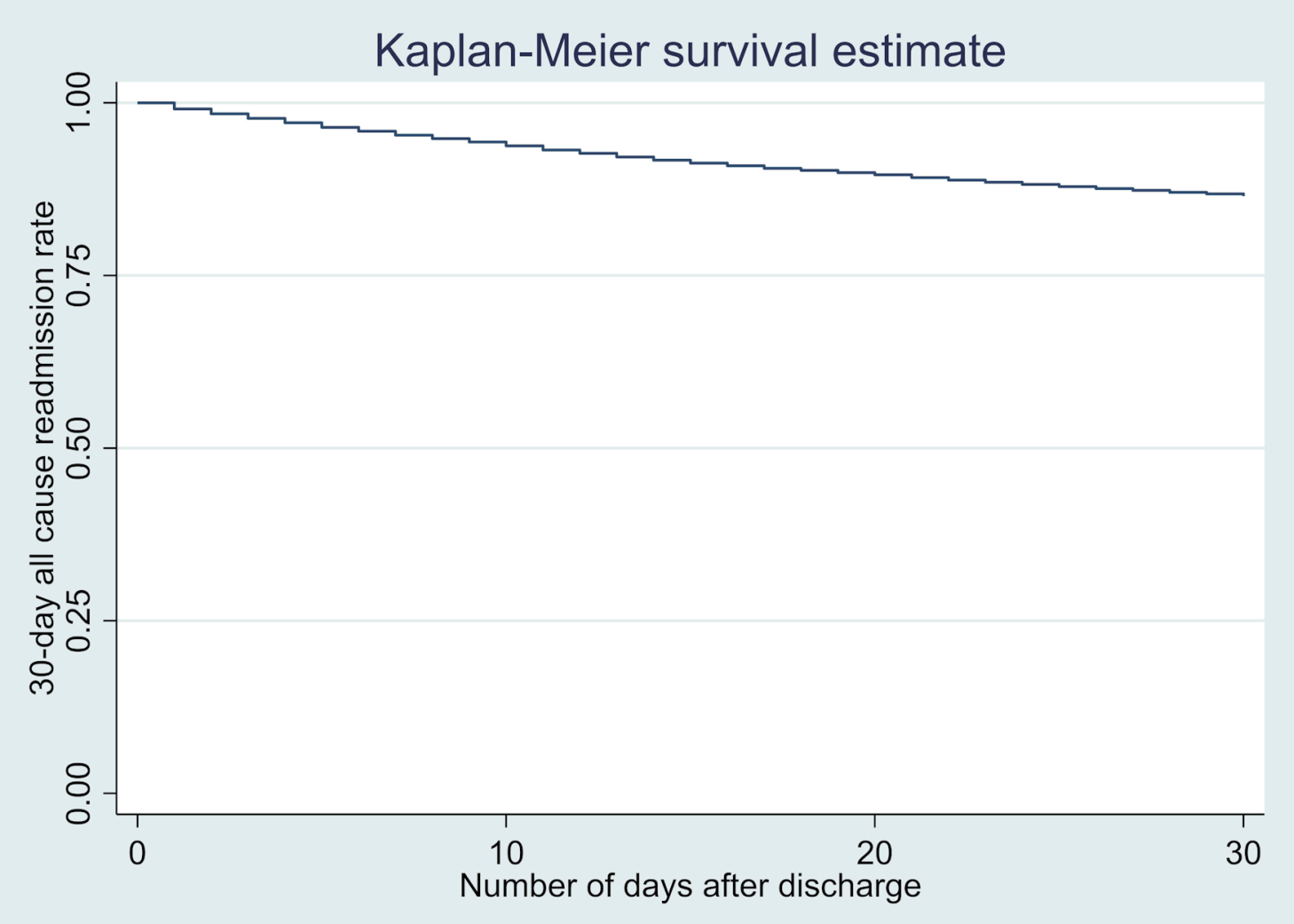

Figure 1. Kaplan-Meier graph for 30-day all-cause readmissions of hypertriglyceridemia-induced acute pancreatitis (HTG-AP) in the USA in 2018.

dex $(\mathrm{CCI})$ scores $\geq 3(48.5 \%$ vs. $33.8 \%, \mathrm{P}<0.001)$ compared to index admissions. Furthermore, for 30-day readmissions of HTG-AP, we also noted higher proportion of patients with congestive heart failure (CHF) $(14.3 \%$ vs. 9.3\%, P < 0.001), chronic kidney disease (CKD) $(20.8 \%$ vs. $15.5 \%, \mathrm{P}<0.001)$, coronary artery disease (CAD) $(22.3 \%$ vs. $27.0 \%, \mathrm{P}<0.001)$, and protein energy malnutrition (PEM) $(13.2 \%$ vs. $4.9 \%, \mathrm{P}<$ 0.001 ), but a lower proportion of patients with hypertension and obesity compared to index admissions (Table 2).

Table 1. Most Common Principal Diagnosis on Readmission for 30-Day Readmissions of Hypertriglyceridemia-Induced Acute Pancreatitis (HTG-AP) in the USA in 2018

\begin{tabular}{ll}
\hline Principal readmission diagnosis $(\mathbf{n}=\mathbf{5 , 7 7 3})$ & Proportion (\%) \\
\hline Acute pancreatitis & 45.2 \\
Chronic pancreatitis & 6.3 \\
Sepsis (unspecified organism) & 4.8 \\
Acute renal failure & 1.6 \\
Malignant neoplasm of head of pancreas & 1.1 \\
Type 2 diabetes mellitus with ketoacidosis & 1.0 \\
Pneumonia (unspecified organism) & 0.9 \\
Hypertensive heart disease with heart failure & 0.7 \\
Non-ST elevation myocardial infarction & 0.7 \\
\hline
\end{tabular}

\section{Outcomes for index and 30-day readmissions of HTG-AP}

For 2018, the odds of inpatient mortality for 30-day readmissions of HTG-AP were higher $(1.7 \%$ vs. $0.7 \%$, odds ratio $(\mathrm{OR})$ : $2.55,95 \%$ confidence interval $(\mathrm{CI}): 1.83-3.57, \mathrm{P}<0.001)$ compared to index admissions. Furthermore, 30-day readmissions of HTG-AP was also associated with longer LOS (5.6 vs. 4.1 days, OR: $1.5,95 \% \mathrm{CI}: 1.2-1.7, \mathrm{P}<0.001)$ and mean THC (\$56,799 vs. \$36,078, OR: 18,702, 95\% CI: 15,136 - 22,267, P $<0.001$ ) compared to index admission (Table 3 ).

\section{Predictors for 30-day readmissions of HTG-AP}

Independent predictors of 30-day all-cause readmissions of HTG-AP included hypertension (adjusted hazard ratio (aHR): 1.12, 95\% CI: $1.01-1.26, \mathrm{P}=0.040), \mathrm{PEM}$ (aHR: 1.96, 95\% CI: $1.68-2.28, \mathrm{P}<0.001), \mathrm{CCI}$ scores $\geq 3$ (aHR: $1.80,95 \% \mathrm{CI}$ : $1.53-2.11, \mathrm{P}<0.001)$, CKD (aHR: $1.29,95 \% \mathrm{CI}: 1.11-1.14$, $\mathrm{P}=0.001)$ and discharge against medical advice (AMA) (aHR: $1.80,95 \%$ CI: $1.44-2.24, \mathrm{P}<0.001$ ) (Table 4).

\section{Discussion}

HTG-AP is currently the third most common cause of AP with 
Table 2. Comparison of Hospitalization Characteristics for Index and 30-Day Readmissions of Hypertriglyceridemia-Induced Acute Pancreatitis (HTG-AP) in the USA in 2018

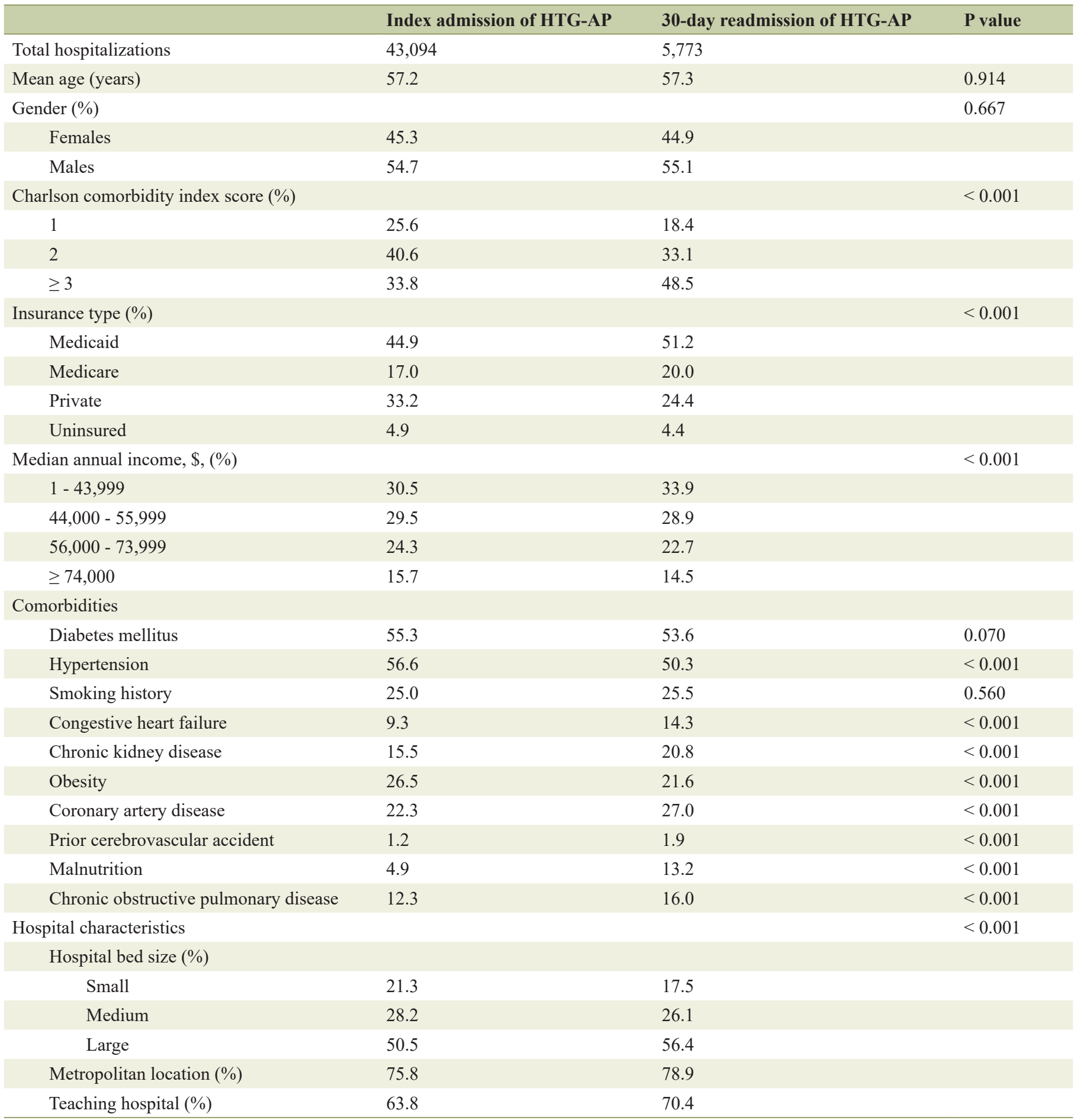

severity ranging from self-limiting pancreatic edema to multiorgan involvement and mortality [15]. Triglyceride levels $>$ $1,000 \mathrm{mg} / \mathrm{dL}$ are usually associated with HTG-AP, but no clear thresholds have been defined in current literature. The exact pathogenic mechanism implicated in the development of HTG-
AP is currently unknown. However, it is postulated that it may be secondary to increased conversion of excessive triglycerides to free fatty acids by pancreatic lipase ultimately leading to pancreatic ischemia and acinar cell injury [5]. Furthermore, patients with HTG-AP have a higher likelihood of progression to severe 
Table 3. Comparison of Clinical Outcomes for Index and 30-Day Readmissions of Hypertriglyceridemia-Induced Acute Pancreatitis (HTG-AP) in the USA in 2018

\begin{tabular}{lllll}
\hline Outcomes & Index admission of HTG-AP & 30-day readmission of HTG-AP & aOR (95\% CI) & P value \\
\hline Inpatient mortality (\%) & 0.7 & 1.7 & $2.55(1.83-3.57)$ & $<0.001$ \\
Mean length of stay (days) & 4.1 & 5.6 & $1.5^{\mathrm{a}}(1.2-1.7)$ & $<0.001$ \\
Mean total hospital charge $(\$)$ & 36,078 & 56,779 & $18,702^{\mathrm{a}}(15,136-22,267)<0.001$ \\
\hline
\end{tabular}

aMean difference. aOR: adjusted odds ratio; $\mathrm{Cl}$ : confidence interval.

disease and prolonged hospital course [16, 17].

In literature, readmission rates for AP have been reported to be as high as 15-29\% [18-20]. An NRD-based study in 2013 reported the 30-day readmission rate of AP to be as high as $16.2 \%[18,19]$. In our study, the 30 -day all-cause readmission rate for HTG-AP was $13.5 \%$, which was in line with current literature. Furthermore, only about half $(45.2 \%)$ of the patients with HTG-AP had a principal diagnosis of AP at presentation on readmission. This finding mirrored prior studies which have reported recurrent attacks of pancreatitis as the most common cause for readmission after an index admission of AP [9]. This reflects the need for aggressive triglyceride control in these patients to decrease overall readmission rates and the burden of the disease on the US healthcare system. From a mortality perspective, 30-day readmissions of HTG-AP had higher odds of inpatient mortality compared to index admissions (Table 3). Therefore, it is vital to appropriately manage index admissions through a multi-disciplinary team approach with antihyperlipidemic agents, insulin, and/or heparin, and promote patient education and encourage regular outpatient follow-up in an attempt to decrease readmissions and inpatient mortality [21].

The presence of associated comorbidities on index admission for any disease negatively impacts the patient's overall health leading to higher risk of complications and exacerbations $[22,23]$. In patients with AP, studies have demonstrated that the presence of advanced CKD, CAD, hypertension, metabolic disorders such as diabetes and malnutrition, and increasing CCI scores are associated with higher odds of early readmissions [8]. Similarly, in our study, 30-day readmissions of HTG-AP had a higher proportion of patients with comorbidities such as CHF, CAD, CKD, and PEM. Additionally, 30day readmissions of HTG-AP also had a high comorbidity burden $(\mathrm{CCI} \geq 3)$ compared to index admissions, which was also an independent predictor for 30-day all-cause readmissions.
These findings reflect that in patients with known comorbidities, an initial episode of AP debilitates overall health thereby increasing risk of readmissions.

We noted a longer LOS (5.6 vs. 4.1 days, OR: 1.5, 95\% CI: $1.2-1.7, \mathrm{P}<0.001$ ) for 30-day readmissions of HTG-AP compared to index admissions. This may be due to the fact these readmissions may have associated complications and a greater degree of severity often requiring prolonged hospitalization. Furthermore, an NRD-based study in 2013 reported attributable hospital charges of approximately $\$ 1.10$ billion and $\$ 3.86$ billion from index and first readmissions of AP, respectively [18]. Our study further underlines these findings as we report significantly higher THC (\$56,799 vs. \$36,078, OR: $18,702,95 \% \mathrm{CI}$ : $15,136-22,267, \mathrm{P}<0.001)$ for 30 -day readmissions of HTG-AP compared to index admissions. The higher costs for 30-day readmissions of HTG-AP may be secondary to a prolonged hospitalization course, higher level of care, and presence of complications often requiring multi-disciplinary sub-specialty care.

In our study, PEM was an independent predictor for 30day all-cause readmissions of HTG-AP. Literature reports that over $62 \%$ of all patients with index hospitalization for AP may have exocrine pancreatic insufficiency (EPI) which can potentially lead to malabsorption [24, 25]. Hence, inability to meet nutritional requirements due to the presence of EPI coupled with an exacerbation of the pain of pancreatitis and/or fear of eating may lead to malnutrition and increase the susceptibility of these individuals to readmissions [26, 27]. Additionally, discharge AMA was another independent predictor for 30-day allcause readmissions of HTG-AP. Studies have reported higher rates of readmissions for patients discharged AMA due to incomplete treatments and lack of discharge instructions, which may have been the case in our cohort [20, 28]. Furthermore, we observed that CKD was not only seen in a higher proportion of 30-day readmissions of HTG-AP, but also served as an

Table 4. Predictors of 30-Day Readmissions of Hypertriglyceridemia-Induced Acute Pancreatitis (HTG-AP) in the USA in 2018

\begin{tabular}{llll}
\hline Variable & aHR & $\mathbf{9 5 \%}$ CI & P value \\
\hline Protein energy malnutrition & 1.96 & $1.68-2.28$ & $<0.001$ \\
Discharge against medical advice & 1.80 & $1.44-2.24$ & $<0.001$ \\
Charlson comorbidity index score $\geq 3$ & 1.80 & $1.53-2.11$ & $<0.001$ \\
Chronic kidney disease & 1.29 & $1.11-1.49$ & 0.001 \\
Hypertension & 1.12 & $1.01-1.26$ & 0.041 \\
Age & 0.99 & $0.98-0.99$ & $<0.001$ \\
Obesity & 0.83 & $0.74-0.93$ & 0.001 \\
\hline
\end{tabular}

aHR: adjusted hazard ratio; $\mathrm{Cl}$ : confidence interval. 
independent predictor of 30-day all-cause readmissions. This may, in part, be secondary to decreased elimination of pancreatic enzymes in patients with CKD coupled with damage to the pancreas due to uremia and dialysis [29].

Our study has several strengths and limitations. One key strength of this study is the study population, which is derived from one of the largest, multiethnic, inpatient databases available in the USA. Additionally, an analysis and comparison of various demographic and outcome-oriented facets for index and 30-day readmissions of HTG-AP offers a comprehensive and thorough overview of the burden of HTG-AP readmissions on individual and the US healthcare system. Moreover, identification of the predictors of all-cause readmissions may help physicians identify individuals at greatest risk of readmission during the index hospitalization. However, we do acknowledge all the limitations associated with our study. The NRD does not contain information on the time of discharge from index admission to readmission, severity of HTG-AP at the time of index admission or readmission, hospital course, laboratory investigations for index admission or readmission, and the pharmacological management of the disease. Due to limitations associated with the NRD database, we were also unable to determine the exact etiology of the underlying HTG or pregnancy status in the study population. Furthermore, due to the retrospective nature of the study, it is subject to all biases associated with retrospective studies. Lastly, the NRD is an administrative database that uses International Classification of Diseases, Tenth Revision/Procedure Coding System ICD-10/PCS codes to store information. Hence, the possibility of human coding errors cannot be excluded. However, despite these limitations, our large sample size, comprehensive analysis technique, and scientific inquires contribute to a wellrounded study which sheds light on a relative underinvestigated topic, while aiming to encourage future research on the topic with large, multi-centered prospective studies.

\section{Conclusions}

HTG is believed to be the third most common cause of AP around the globe. The pathogenic mechanism implicated in the development of HTG-AP is poorly understood; however, it is associated with significant morbidity, mortality and healthcare burden. Additionally, due to poor triglyceride control, these individuals are at risk of readmissions. In our study, the 30-day all-cause readmission rate for HTG-AP was noted to be $13.5 \%$. AP was identified as the most common principal diagnosis at the time of readmission, followed by chronic pancreatitis and unspecified sepsis. After a comparative analysis, 30-day readmissions of HTG-AP had a higher comorbidity burden (CCI $\geq$ 3 ), inpatient mortality, mean LOS and mean THC compared to index admissions. Furthermore, hypertension, PEM, CCI scores $\geq 3, \mathrm{CKD}$, and discharge AMA were identified as independent predictors for 30-day all-cause readmissions of HTG-AP.

\section{Acknowledgments}

None to declare.

\section{Financial Disclosure}

The authors have no financial disclosure.

\section{Conflict of Interest}

The authors report no conflict of interest.

\section{Informed Consent}

As the NRD database lacks patient-specific identifiers, no consent was required for the analysis.

\section{Author Contributions}

Substantial contributions to the conception and design of the work: Asim Kichloo, Zain El-amir, and Dushyant Singh Dahiya. Acquisition, analysis, or interpretation of data for the work: all authors. Literature search and review: all authors. Drafting the work: all authors. Revising it critically for important intellectual content: Dushyant Singh Dahiya, Mohammad Al-Haddad, Sailaja Pisipati and Asim Kichloo. Final approval of the version to be published: all authors. Agreement to be accountable for all aspects of the work: all authors.

\section{Data Availability}

The data supporting the findings of this study is publicly available at https://www.hcup-us.ahrq.gov.

\section{Abbreviations}

AHRQ: Agency for Healthcare Research and Quality; AMA: against medical advice; AP: acute pancreatitis; CAD: coronary artery disease; CCI: Charlson comorbidity index; CHF: congestive heart failure; CKD: chronic kidney disease; HCUP: Healthcare Cost and Utilization Project; HTG: hypertriglyceridemia; HTG-AP: hypertriglyceridemia-induced acute pancreatitis; ICD-10-CM/PCS: International Classification of Diseases, Tenth Revision, Clinical Modification/Procedure Coding System; IRB: Institutional Review Board; LOS: length of stay; NRD: National Readmission Database; PEM: protein energy malnutrition; THC: total hospital charge

\section{References}

1. Dron JS, Wang J, Cao H, McIntyre AD, Iacocca MA, Menard JR, Movsesyan I, et al. Severe hypertriglyceridemia is primarily polygenic. J Clin Lipidol. 2019;13(1):80-88.

2. Lee PJ, Papachristou GI. New insights into acute pancreatitis. Nat Rev Gastroenterol Hepatol. 2019;16(8):479- 
496.

3. Crockett SD, Wani S, Gardner TB, Falck-Ytter Y, Barkun AN, American Gastroenterological Association Institute Clinical Guidelines Committee. American Gastroenterological Association Institute Guideline on Initial Management of Acute Pancreatitis. Gastroenterology. 2018;154(4):1096-1101.

4. Valdivielso P, Ramirez-Bueno A, Ewald N. Current knowledge of hypertriglyceridemic pancreatitis. Eur J Intern Med. 2014;25(8):689-694.

5. Garg R, Rustagi T. Management of hypertriglyceridemia induced acute pancreatitis. Biomed Res Int. 2018;2018:4721357.

6. Boxhoorn L, Voermans RP, Bouwense SA, Bruno MJ, Verdonk RC, Boermeester MA, van Santvoort HC, et al. Acute pancreatitis. Lancet. 2020;396(10252):726-734.

7. Peery AF, Crockett SD, Barritt AS, Dellon ES, Eluri S, Gangarosa LM, Jensen ET, et al. Burden of gastrointestinal, liver, and pancreatic diseases in the United States. Gastroenterology. 2015;149(7):1731-1741.e3.

8. Bolourani S, Diao L, Thompson DA, Wang P, Coppa GF, Patel VM, Giangola MD. Risk factors for early readmission after acute pancreatitis: importance of timely interventions. J Surg Res. 2020;252:96-106.

9. Vipperla K, Papachristou GI, Easler J, Muddana V, Slivka A, Whitcomb DC, Yadav D. Risk of and factors associated with readmission after a sentinel attack of acute pancreatitis. Clin Gastroenterol Hepatol. 2014;12(11):19111919.

10. HCUP Nationwide Readmission Database (NRD). 2018. Healthcare Cost and Utilization Project (HCUP). Agency for Healthcare Research and Quality, Rockville, MD. https://www.hcup-us.ahrq.gov/nrdoverview.jsp. Accessed: October 15th, 2021.

11. Fortson MR, Freedman SN, Webster PD, 3rd. Clinical assessment of hyperlipidemic pancreatitis. Am J Gastroenterol. 1995;90(12):2134-2139.

12. Shaka H, Asotibe JC, Achebe I, Pudasaini G. Higher inpatient morbidity and mortality in biliary pancreatitis compared to hypertriglyceridemia-induced pancreatitis: a nationwide retrospective study. Cureus. 2020;12(9):e10351.

13. Sundararajan V, Quan H, Halfon P, Fushimi K, Luthi JC, Burnand B, Ghali WA, et al. Cross-national comparative performance of three versions of the ICD-10 Charlson index. Med Care. 2007;45(12):1210-1215.

14. HCUP Cost-to-Charge Ratio Files (CCR). Healthcare Cost and Utilization Project (HCUP). 2018. Agency for Healthcare Research and Quality, Rockville, MD. www. hcupus.ahrq.gov/db/ccr/costtocharge.jsp. Accessed: October 15th, 2021.

15. Goodchild G, Chouhan M, Johnson GJ. Practical guide to the management of acute pancreatitis. Frontline Gastro- enterol. 2019;10(3):292-299.

16. Yang AL, McNabb-Baltar J. Hypertriglyceridemia and acute pancreatitis. Pancreatology. 2020;20(5):795-800.

17. Yan P, Zhao HX, Chen X. Suboptimal management of hypertriglyceridemia in the outpatient setting is associated with the recurrent pancreatitis: A retrospective cohort study. Medicine (Baltimore). 2020;99(43):e22887.

18. Munigala S, Subramaniam D, Subramaniam DP, Buchanan P, Xian H, Burroughs T, Trikudanathan G. Predictors for early readmission in acute pancreatitis (AP) in the United States (US) - A nationwide population based study. Pancreatology. 2017;17(4):534-542.

19. Garg SK, Campbell JP, Anugwom C, Wadhwa V, Singh R, Gupta N, Sanaka MR. Incidence and predictors of readmissions in acute pancreatitis: a nationwide analysis. Pancreas. 2018;47(1):46-54.

20. Argueta PP, Salazar M, Vohra I, Corral JE, Lukens FJ, Vargo JJ, Chahal P, et al. Thirty-Day Readmission Among Patients with Alcoholic Acute Pancreatitis. Dig Dis Sci. 2021;66(12):4227-4236.

21. de Pretis N, Amodio A, Frulloni L. Hypertriglyceridemic pancreatitis: Epidemiology, pathophysiology and clinical management. United European Gastroenterol J. 2018;6(5):649-655.

22. Donze J, Lipsitz S, Bates DW, Schnipper JL. Causes and patterns of readmissions in patients with common comorbidities: retrospective cohort study. BMJ. 2013;347:f7171.

23. Yalin SF, Bakir A, Trabulus S, Seyahi N, Altiparmak MR. The Charlson Comorbidity Index: can it predict the outcome in acute kidney injury? Int Urol Nephrol. 2020;52(9):1713-1718.

24. Dominguez-Munoz JE. Pancreatic exocrine insufficiency: diagnosis and treatment. J Gastroenterol Hepatol. 2011;26(Suppl 2):12-16.

25. Huang W, de la Iglesia-Garcia D, Baston-Rey I, CalvinoSuarez C, Larino-Noia J, Iglesias-Garcia J, Shi N, et al. Exocrine pancreatic insufficiency following acute pancreatitis: systematic review and meta-analysis. Dig Dis Sci. 2019;64(7):1985-2005.

26. Mergener K, Baillie J. Chronic pancreatitis. Lancet. 1997;350(9088):1379-1385.

27. Roberts KM, Nahikian-Nelms M, Ukleja A, Lara LF. Nutritional Aspects of Acute Pancreatitis. Gastroenterol Clin North Am. 2018;47(1):77-94.

28. Garland A, Ramsey CD, Fransoo R, Olafson K, Chateau D, Yogendran M, Kraut A. Rates of readmission and death associated with leaving hospital against medical advice: a population-based study. CMAJ. 2013;185(14):12071214.

29. Golay V, Roychowdhary A. Acute pancreatitis in chronic kidney disease - a common but often misunderstood combination. Ren Fail. 2012;34(10):1338-1340. 